\title{
SEAMLESS MOSAIC OF UAV IMAGES FOR DENSE URBAN AREA
}

\author{
Ming $\mathrm{Li}^{1,2}$, Ruizhi Chen ${ }^{1,2}$, Xuan Liao ${ }^{1}$ and Weilong Zhang ${ }^{1}$ \\ ${ }^{1}$ State Key Laboratory of Information Engineering in Surveying Mapping and \\ Remote Sensing, Wuhan University, Wuhan City, China \\ ${ }^{2}$ Collaborative Innovation Center of Geospatial Technology, \\ Wuhan University, Wuhan City, China
}

\begin{abstract}
This paper aims to put forward a seamless mosaic method of UAV image for dense urban area, which can effectively avoid seam-line pass through the edge of the building, so as to eliminate the ghosting, dislocation and seam in the image mosaic process. Firstly, the radiation error of UAV image are corrected by Wallis algorithm, and extract the corresponding points from the adjacent images by SIFT algorithm, to correct the left and right pending matching images to the virtual unified reference image, to ensure the images are in the same coordinate system. Then, in view of the shortcomings of the classical Duplaquet method, we proposed a new more robust UAV image mosaic algorithm by changing the energy accumulation criterion of energy function for dynamic programming. Finally, the comparative experiments show that our method can find the optimal seam-line to avoid it through the edge of houses, especially in dense urban area.
\end{abstract}

\section{KEYWORDS}

UAV Images, Seamless Mosaic, Seam-line, Dynamic Programming, Dense Urban Area

\section{INTRODUCTION}

Unmanned aerial vehicles (UAV) remote sensing system has been widely used in environmental protection, ecological agriculture, disaster emergency and 3D urban reconstruction with its predominance of low cost, fast and easy to operation. However, UAV due to flight height, camera perspective constrains, the coverage of single UAV image is small. In order to expand the field of view, obtain more remote sensing information about the target area, we need to mosaic multiple UAV images into one image. Because of UAV is lighter in quality, vulnerable to high-altitude winds, flight attitude is unstable, the overlapping area of adjacent images is often irregular, and the image exposure is uneven, they always lead to stitching images prone to ghosting, blurred, dislocation, colour inconsistencies and so on ${ }^{[1-5]}$.

Internationally, researchers have proposed a lot of methods to solve the problem of seamless mosaic of UAV images. Among them, seam-line-based algorithms are an important research branch in this area. This kind of algorithms are intended to find an optimal path with less grayscale and geometric differences, energy function-based algorithm is one of the important methods to resolve the problem. It focus on considering the energy difference between the images, and its effect is more superior to others. In the energy function-based algorithms, references [6-8] adopt the Dijkstra's shortest path algorithm to search for the optimal seam-line, which improves the ghosting and dislocation problems due to objects movement, registration

David C. Wyld et al. (Eds) : CNDC, DPPR, AIAA, WiMNET, ACITY - 2017

pp. 33- 39, 2017. (C) CS \& IT-CSCP 2017

DOI : $10.5121 /$ csit.2017.71604 
errors, but its search efficiency is low, and the method of weight determination is complex too. The seam-line searching by the ant colony algorithm can avoid the area where the colour contrast is larger on the image, but the algorithm is sensitive to the number of ants, this will causes the search process of seam-line easy to fall into the local optimum ${ }^{[9]}$. However, the dynamic programming algorithm is relatively mature, has a relatively complete theoretical system, not easy to innovation of it, and after modified the energy function of dynamic programming algorithm, it still has a strong image direction correlation, that leads to reduce its robustness, especially there is a significant difference in brightness between the adjacent images. Most of the current algorithms still cannot achieve satisfactory results when dealing with image mosaics in dense urban areas. Therefore, this paper attempts to propose a new UAV image mosaic method based on dynamic programming for dense urban area.

\section{IMPROVED IMAGE MOSAIC METHOD}

\subsection{Classic method of dynamic programming}

The Duplaquet method is a classic dynamic programming method for seam-line searching. Formula (1) is the energy criterion defined for the algorithm ${ }^{[10]}$. The algorithm can ensure that the length of the alternative seam-lines are equal, and the seam-line with the smallest accumulated energy values is the optimal.

$$
C(x, y)=C_{\text {dif }}(x, y)-\lambda C_{\text {edge }}(x, y)
$$

Thereinto, $\mathrm{C}_{d i f}(x, y)$ is the mean value of the grey level difference of the pixel in the neighbourhood $V$ which belong to the overlapping areas between the two adjacent images, $\mathrm{C}_{\text {edge }}$ $(x, y)$ is the minimum gradient value of the pixel in the overlapping areas of image pair, $\lambda$ is a weighing factor, which be used for adjusting the proportion of grey difference and structure difference in energy function. There are many improved methods. Reference [11] is a representative improvement method based on Duplaquet algorithm, the method put the ratio value of accumulate energy value and the length of seam-line as a measurement criteria, and took the smallest value as the best one, but it still has some problems. It can be seen from the Figure 1, compared with the Duplaquet method, its best seam-line also still pass through a large number of houses, this is not conducive to UAV image mosaic in dense urban areas.
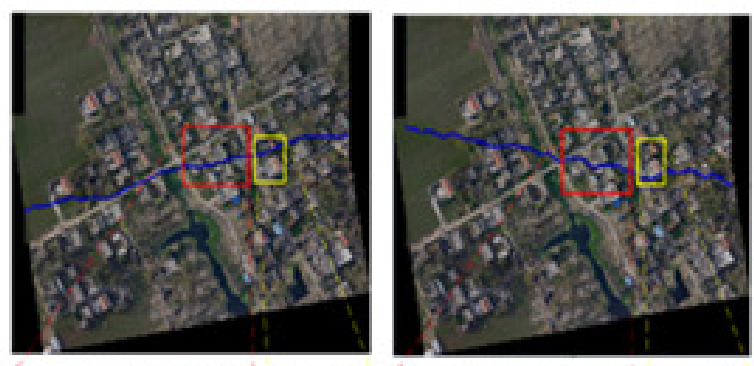

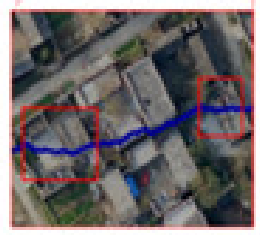

(a)
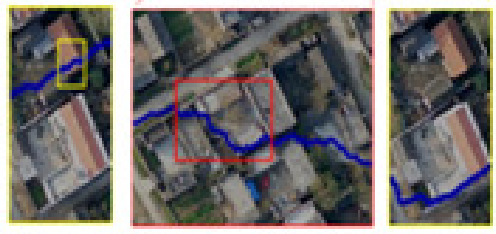

(b)

Figure 1. Seam-lines by different methods. (a) Duplaquet method; (b) Reference [11] Method 


\subsection{Our improved method}

The methods mentioned above have the following two problem: 1) Gradient guidance direction of energy function does not support omnidirectional searching. 2) The accumulation of energy function has directionality. The optimal energy function aggregation takes only three directions (energy aggregation directions) into consideration, and the direction of energy aggregation is also limited from left to right, from top to bottom direction (energy traversal direction). The energy criterion proposed by the classic Duplaquet algorithm only considers the horizontal and vertical gradients, and only compares the pixels in the three adjacent directions near the current pixel. When the overlapping area has a large number of dense distribution of buildings, and these houses with different height, due to the deformation are not consistent from image point to the ground point in roof, the seam-lines searched by the Duplaquet algorithm are likely to pass through the edge of the buildings, and when the image matching errors are large, the stitched image is easy to appear obvious dislocation phenomenon.

In order to solve these problems. Firstly, on the basis of considering the 8 directional neighbourhood information of current pixel and its similarity of surrounding structure, we use a new operator to calculate the pixel gradients according to reference [12]. Then, we introduce the fourth direction on the basis of the original three direction in the process of seam-line searching, to correct the problem of seam-line serious deviation from the ideal seam-line by changing the strategy of energy aggregation direction. At last, on the foundation of the Duplaquet method, we redefined the energy criterion and proposed a new dynamic programming method based on twochannel energy accumulation to improve the optimal seam-line searching. As shown in Figure 2, there is a schematic diagram of two-channel-based optimal seam-line searching, which optimizes the seam-line searching criteria by detecting the eight pixels (contain the horizontal direction) that surrounding the current pixel neighbourhood.
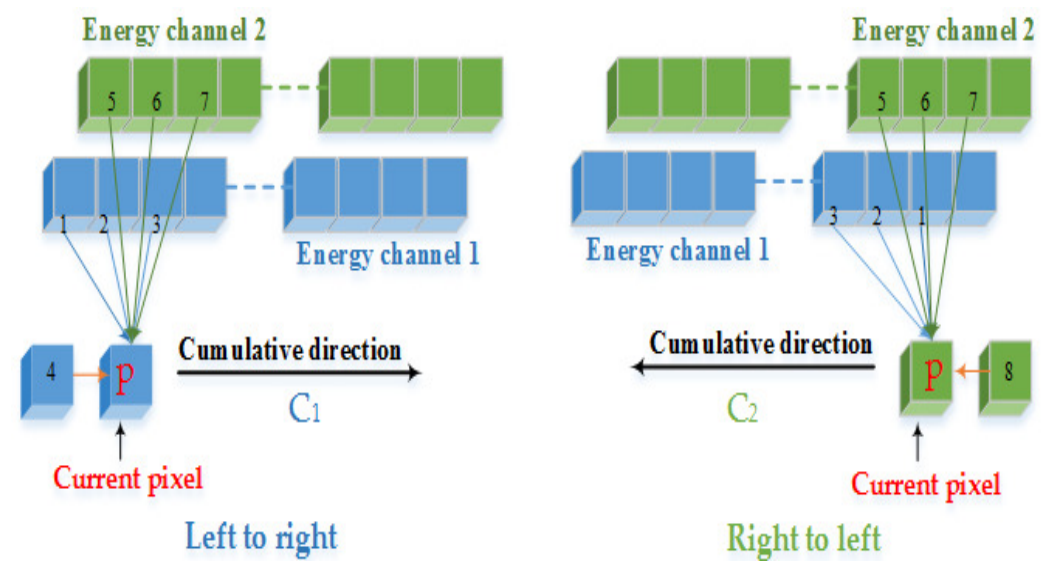

Figure 2. The idea of our improved method

In Figure 2, set $\mathrm{P}$ as the current pixel, and redefine the eight comparison directions of $\mathrm{P}$, which respectively is 1(upper left of the current pixel for energy aggregation channel 1), 2(upper of the current pixel for energy aggregation channel 1), 3(upper right of the current pixel for energy aggregation channel 1), 4(left of the current pixel for energy aggregation channel 1), 5(upper left of the current pixel for energy aggregation channel 2), 6(upper of the current pixel for energy aggregation channel 2), 7(upper right of the current pixel for energy aggregation channel 2), 8 (right of the current pixel for energy aggregation channel 2), the initial invalid direction is the current pixel itself. 
Based on the theoretical analysis, this paper constructed the mathematical abstract expression of the theoretical model that proposed in this paper. Assuming that image 1 and image 2 are original image pair be used for stitching, the energy function defined in this paper is shown in formula (2):

$$
\begin{aligned}
& E=\sum_{(x, y) \in O} B(x, y) \sigma\left(\left|I_{1}(x, y)-I_{2}(x, y)\right|\right)+ \\
& \sum_{(x, y) \in O} \sigma\left(\max \left(\left|\underset{0 \leq k \leq 7}{d_{k}}\left(I_{1}(x, y)\right)-{\underset{0 \leq k \leq 7}{k}}_{0}\left(I_{2}(x, y)\right)\right|\right)\right)+\sum_{(x, y) \notin O} N(x, y)
\end{aligned}
$$

In formulation (2), $B(x, y)$ to determine whether the current pixel $(x, y)$ is in the boundary of overlapping area of the adjacent images, when $B(x, y)=1$, it means that it is not in the boundary region, when $B(x, y)=10$, it means that it is in the boundary region. $\sigma_{(*)}$ is the Gaussian smoothing term, which uses the information in the local window to enhance the local influence of the current pixel, $I_{1}(\cdot), I_{2}(\cdot)$ respectively is the pending stitching image, $O$ is the overlapping area, $d(*)$ represents the gradient function of one of the eight directions, $N(x, y)$ is the energy value of the invalid area, which is the constant term, and the value is 100 times than the maximum value of $O$. If the overlapping area is irregular, it can be extended to a regular area using the smallest circumscribed rectangle of the overlapping area.

\section{EXPERIMENT RESULTS ANALYSIS}

\subsection{Experimental data and environment}

In order to verify the effectiveness of our proposed method, this paper selects the UAV image from dense urban area to testing. And compares the method proposed in this paper with the classic Duplaquet method. Before seam-line searching, the radiation errors of UAV images are corrected by Wallis ${ }^{[13]}$, and the pending mosaic images extracted the corresponding points by SIFT ${ }^{[5]}$ to correct them to a same virtual unified coordinate system. In this paper, it use Visual $\mathrm{C}++$ based on OpenCV open source library to program the proposed improvement algorithm. Figure 3 is two group of experimental images. The experimental computer environment is Windows 7 operating system, with $32 \mathrm{G}$ computer memory, Intel core 7 CPU.

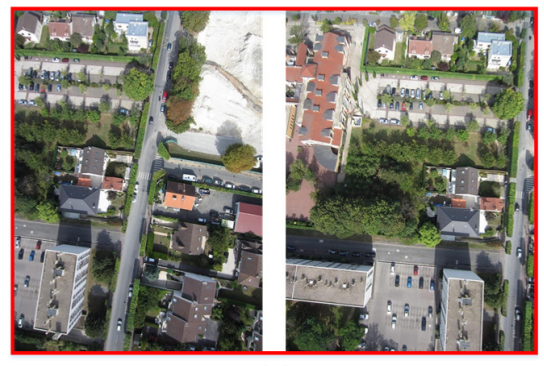

(a)

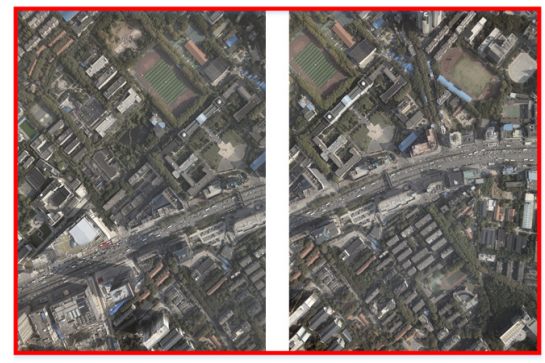

(b)

Figure 3. The experimental data. (a) Data 1; (b) Data 2 


\subsection{Results analysis}
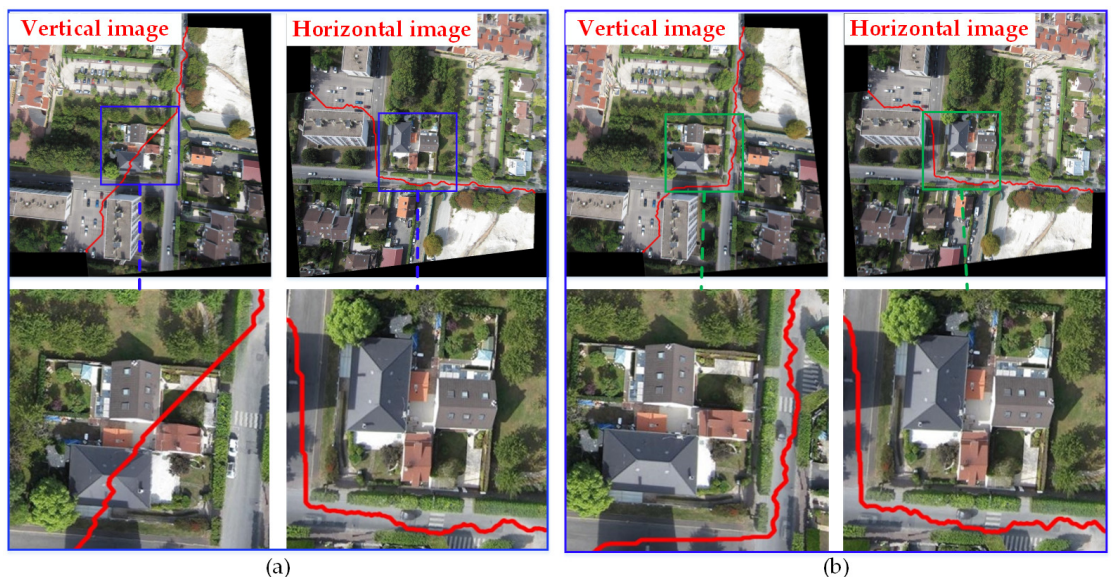

Figure 4. The seam-lines of two methods under rotation. (a) Duplaquet method; (b) our method

Firstly, the vertical image in Figure 3(a) was rotated to be horizontal image, and then, we used the Duplaquet method and the method proposed in this paper to get the seam-lines. Figure 4 shows the results, it can be seen from the pictures of partial enlargement that the best seam-lines searched by the Duplaquet method has changed significantly before and after rotation, the seamline pass through the edge of buildings before rotation, but the seam line avoid the buildings after rotation. The seam-lines are searched by our algorithm are basically no change before and after rotation, they still along the direction of the road forward, and are very good to avoid the ground buildings. This shows that the traditional methods are sensitive to the direction of images, that is to say the minimum value of energy function is related to the direction of energy aggregation and traversal. Therefore, due to this paper has made specific improvements to the above issues, our algorithm has the advantage of adaptability, and it is more robust. So, it is more suitable to the UAV images mosaicking.

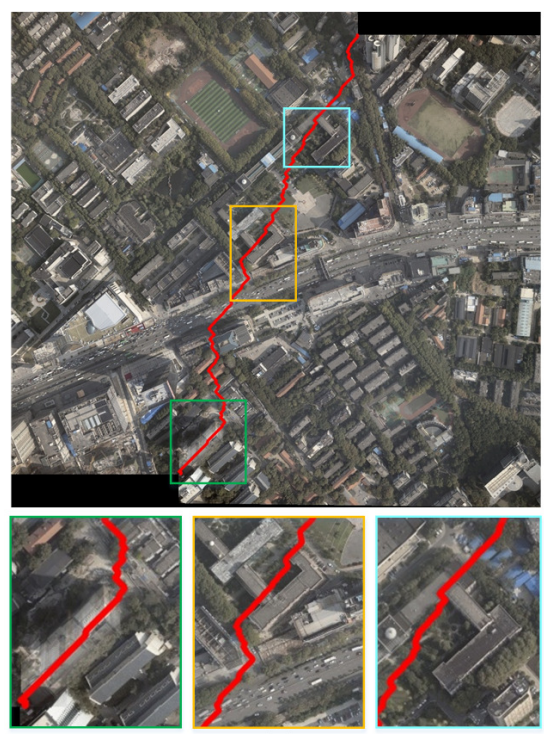

(a)

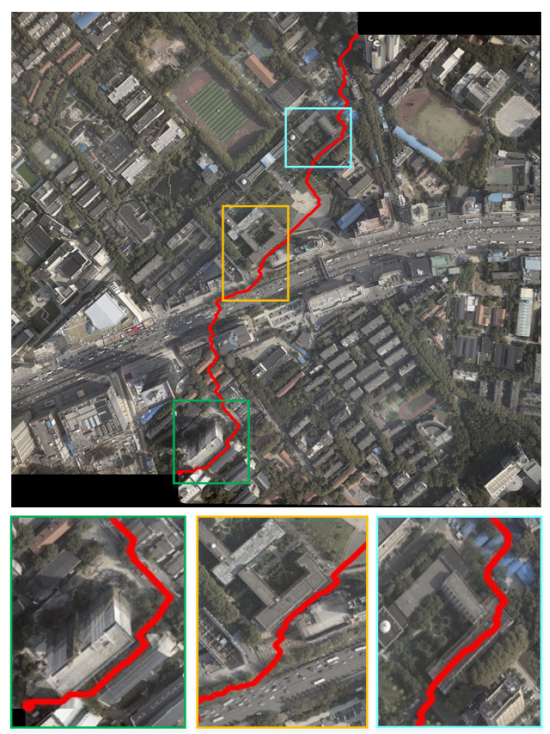

(b)

Figure 5. Compared with seam-lines. (a) Duplaquet method; (b) our method

In order to further verify the superiority of our method, the Duplaquet algorithm and our algorithm are used to search the best seam-lines of image pairs in Figure 3(b) with irregular 
overlapping areas. Figure 5(a) and 5(b) are the results respectively. It can be seen from Figure 5 that the seam-lines of two data sets have obviously difference with different methods. From the local zoom view of Figure 5, we can find that the Duplaquet method not only appear the seamlines across the edge of houses, but also there is a ghosting phenomenon appears in Figure 5(a). In this paper, the optimal seam-lines searched by ours are basically forward along the road direction, which avoid across the ground buildings, it will greatly reduce the probability of dislocation and seams as the reason like image matching errors.

\section{CONCLUSIONS}

This paper reviewed many mainstream image mosaic methods at first, then selected the classical Duplaquet method as the research object, and pointed out the defects of the dynamic programming method based on the Duplaquet algorithm theory. At last, this paper proposed a new dynamic programming algorithm to search the best seam-lines through improved several essential and key problems of the classic Duplaquet method. And furthermore, the superiority and effectiveness of the method proposed in this paper are verified by the comparative experiments of two image pairs with irregular overlapping area. The results are better than the Duplaquet method, and it is proved to be directional independent and better robustness.

\section{ACKNOWLEDGEMENTS}

This Study is supported by the National Key Research and development Program of China (2016YFB0502201), the NSFC (91638203), the State Key Laboratory Research Expenses of LIESMARS.

\section{REFERENCES}

[1] LI, D. \& Li, M., (2014) "Research advance and application prospect of unmanned aerial vehicle remote sensing system", Geomatics and Information Science of Wuhan University. Vol. 39, No. 5, pp505-513.

[2] Chen, R.; Chu, T.; Landivar, J.; Yang, C. \& Maeda, M., (2017) "Monitoring cotton (Gossypium hirsutum L.) germination using ultrahigh-resolution UAS images”, Precision Agric. Vol.18, pp 1-17.

[3] Chen, S.; Lafer, D.; Mangina, E., (2016) "State of Technology Review of Civilian UAVs", Recent Patents on Engineering, 2016, 10(3): 160-174.

[4] Zhang, W.; Li, M.; Guo, B.; Li, D. \& Guo, G., (2017) "Rapid texture optimization of threedimensional urban model based on oblique images", Sensors. Vol.17, No. 4, pp911-916.

[5] Li, M.; Li, D. \& Fan D., (2012) "A study on automatic UAV image mosaic method for paroxysmal disaster", International archives of the photogrammetry, remote sensing and spatial information science. Vol. B6, pp123-128.

[6] Dijkstra, E., (1995) "A note on two problems in connexion with graphs", Numerische Mathematic. Vol. 1, No. 1, pp269-271.

[7] Davis, J., (1998) “ Mosaics of scenes with moving objects”, IEEE Computer Society Conference on Computer Vision \& Pattern Recognition, Santa Barbara, USA.

[8] Chon, J.; Kim, H. \& Lin, C.,(2010) "Seam-line determination for image mosaicking: A mismatch and the global cost", ISPRS Journal of Photogrammetry and Remote Sensing, Vol. 65, No. 1, pp86-92. 
[9] Zhang, J.; Sun, M. \& Zhang, Z., (2010) "Automated seamline detection for orthophoto mosaicking based on ant colony algorithm", Geomatics and Information Science of Wuhan University. Vol. 34, No. 6, pp675-678.

[10] Duplaquet, L., (1998) "Building large images mosaics with invisible seam-lines", Proceedings of SPIE, Vol. 3387, pp369-377.

[11] Xu, Y.; Xing, C. \& Chen, X., (2011) “A mosaicking method for UAV sequence images based on seam line", Geomatics and information science of Wuhan University, Vol. 36, No. 11, pp1265-1269.

[12] Cheng, X., (2011) "Research on fast produce of orthophoto with UAV sequence images", Wuhan University.

[13] Luo, S., (2015) "Improved dodging algorithm based on Wallis principle". Geomatics Science and Technology. Vol. 3, pp51-58.

\section{AUTHORS}

Ming Li received the Ph.D. degree from Wuhan University, Hubei, China in 2016. He is currently a postdoc researcher at LIESMARS, Wuhan University and an assistant researcher at Collaborative Innovation Center of Geospatial Technology. His research interest includes computer vision, indoor positioning and geographic information application.

Ruizhi Chen received the Ph.D. degree from University of Helsinki, Finland in 1991. He is currently a professor at LIESMARS, Wuhan University and a researcher at Collaborative Innovation Center of Geospatial Technology. His research interest includes computer vision, indoor positioning and so on.

Xuan Liao received the B.S. degree in GIS form China University of Petroleum, Qingdao, Shandong province, China, in 2017. Currently she is pursing the Master degree in Wuhan University, Wuhan, Hubei province, China. Her primary research interest is the application of Remote Sensing and Computer Vision.

Weilong Zhang received the M.S. degree from Xidian University, Shanxi, China in 2015. He is pursing Ph.D. degree in Wuhan University, Wuhan, Hubei province, China. His research interest includes computer vision, photogrammetric survey.
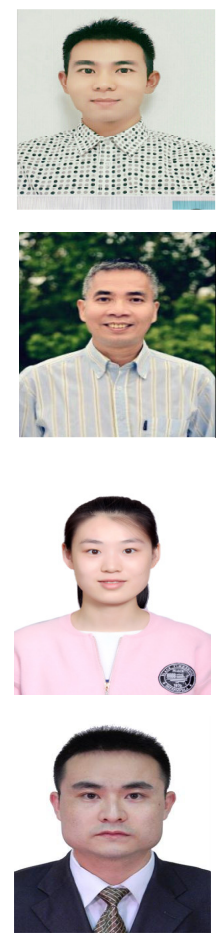